in the Swedish workforce. Future research should continue to assess sex-stratified relationships, using detailed shift work exposure categories and objective registry data where possible.

\section{SHIFTWORK AND CANCER}

Eugene Waclawski. Occupational Health, University of Edinburgh, UK

\subsection{6/oemed-2018-ICOHabstracts. 1374}

Aim of special session What practical advice can Occupational health practitioners give to those at risk and to management for employees:

a. with no obvious risk factors for cancer other than shiftwork,

b. for those with risk factors (strong FHx, gene markers of susceptibility, obesity, benign breast disease, HRT, late menopause, early menarche, late first birth... for breast cancer),

c. those with cancers under treatment or in remission in relation to nightwork,

Also is there a threshold effect? (years of exposure, number of shifts, hours of nightwork),

Is there any need for concern or preventive measures?:

- Should shift lengths be shorter?;

- Should rest breaks should be included; and

- how should researchers educate shift workers and employers as to how sleep-wake cycles are controlled and

- How can we maximise sleep quality, sleep duration, and alertness at work?

Speakers Prof Eva Schernhammer, Associate Professor, Harvard School of Public Health, Boston, USA,

Professor Johnni Hansen Danish Cancer Registry; johnni@cancer.dk.

Prof Lin Fritschi, Curtain University Australia/lin.fritschi@curtin.edu.au.

Prof Damien McElvenny IOM UK; Damien.McElvenny@iom-world.org.

Prof Shelly Tse Lap Ah, Associate Professor, Centre for Occupational and Environmental Health Studies (COEHS), The Chinese University of Hong Kong/shelly@cuhk.edu.hk

\section{6a CAN WE REDUCE HARM FROM SHIFTWORK?}

Lin Fritschi. Curtin University, Perth, Australia

\subsection{6/oemed-2018-ICOHabstracts. 1375}

The association between shiftwork and cancer has been the focus of a number of case-control and cohort studies since the 2010 declaration by the International Agency for Research on Cancer that shiftwork which involves circadian disruption probably caused breast cancer. There is still debate about the association. However shiftwork has also been associated with a range of other health conditions including fatigue, injury, diabetes and cardiovascular disease. In addition, there has been a lot of interest in whether people with different chronotypes (e.g. morning people versus evening people) react to shiftwork differently. Shiftwork cannot be avoided in our 24 hour society, but we have a responsibility to minimise the health impacts upon shiftworkers. We need to consider what aspects of shiftwork might be related to the different health conditions in order to minimise any effect on workers. Thus, whether shiftwork causes breast cancer or not, there is still useful advice we can give shiftworkers in order to improve the quality of life of those working night shifts. This talk will review some of the potential interventions to improve the health for healthier shift work including sleep hygiene, physical activity, food intake, and control of light types and levels.

\section{6b NIGHT SHIFT-WORK AND BREAST CANCER RISK - WHAT IS THE EVIDENCE?}

Johnni Hansen*. Danish Cancer Society Research Centre, Copenhagen Denmark

\subsection{6/oemed-2018-ICOHabstracts. 1376}

Introduction Night work is increasingly common in the modern world. In 2007, the International Agency for Research on Cancer (IARC) classified shift work that involves circadian disruption as probably carcinogenic to humans based on limited evidence from eight epidemiologic studies on breast cancer, in addition to sufficient evidence from animal experiments. The overall evidence was further supported by increased risk in studies of flight attendants, who may be exposed to both shift-work and jet-lag, though night work was not the primary focus in these studies.

Methods Based on a critical review of the scientific literature it is the aim of to present the current epidemiologic evidence and to identify eventual threshold effects, e.g. by duration of night shifts, number of consecutive shifts and menopausal status.

Results After 2007, over 18 new studies with focus on night work and breast cancer are published, which more than triples the number of studies focusing on this issue since the IARC evaluation. The assessment of night shift work is different in all of the studies, which may attenuate the observed increased risk and hinders meta-analysis. There is some evidence that long duration and or high number of consecutive night shifts has impact on the magnitude of increased breast cancer risk. There is also some evidence that increased risk is primarily observed in women who have had nigh work in young adulthood, and in pre-menopausal women.

Conclusion Overall, there is a tendency of increased risk of breast cancer after long-term night shift or after shorter periods with many consecutive shifts. More studies using harmonised definitions of night work metrics is needed. Finally, evidence based preventive interventions are warranted.

\section{C NIGHTSHIFT WORK AND PROSTATE CANCER AMONG HONG KONG CHINESE MEN}

${ }^{1}$ Lap Ah Tse ${ }^{*},{ }^{1}$ Feng Wang, ${ }^{1}$ Priscilla Ming Yi Lee, ${ }^{2}$ Wing Ming Ho, ${ }^{3}$ Chi Fai Ng. ${ }^{1}$ Division of Occupational and Environmental Health, JC School of Public Health and Primary Care, The Chinese University of Hong Kong, Hong Kong SAR, China; ${ }^{2}$ Department of Clinical Oncology, Prince of Wales Hospital, Hong Kong SAR, China; ${ }^{3}$ SH Ho Urology Centre, Department of Surgery, The Chinese University of Hong Kong, Hong Kong SAR, China

\subsection{6/oemed-2018-ICOHabstracts. 1377}

Introduction A positive association between nightshift work and prostate cancer risk has been reported in epidemiological studies, but the findings have been mixed. Also, none of the previous studies has attempted to sufficiently consider the possible confounding effect from dietary sources including 
environmental exposure to bisphenol A (BPA). This study aims to examine the association between nightshift work and prostate cancer risk among Hong Kong Chinese men after take into account more environmental exposures.

Methods We consecutively recruited 431 incident prostate cancer cases and age frequency matched 402 controls who had complete information on nightshift work. After receiving written consents, trained researchers interviewed participants using a standard questionnaire to obtain information on socio-demographics, smoking, dietary habits, habits of using plastic food containers, family cancer history, and occupational history and nightshift work. A newly developed novel cumulative BPA exposure index (CBPAI) was used to estimate chronic BPA exposure. Odds ratio and 95\% confidence interval (95\% CI) was performed using multiple logistic regression analysis.

Results The mean age of prostate cancer cases was comparable to the controls (69.4 vs 68.2 years). Compared with the controls (39.1\%), more cases were less educated with a higher proportion of 'primary school or below' (41.1\%). More cases than controls were the nightshift workers $(13.5 \%$ vs $7.5 \%)$. After adjustment of age and socioeconomic characteristics, the OR of nightshift work to prostate cancer was 1.87 (95\% CI: 1.16 to 3.01$)$, and the OR retained statistical significance $(\mathrm{OR}=1.76,95 \% \mathrm{CI}: 1.07$ to 2.89$)$ after environmental exposures mainly from dietary sources were further adjusted.

Conclusions Results from this study provided supportive evidence that there might be a link between nightshift work and prostate cancer. The main merit of this study is that more environmental risk factors were considered in quantifying the association.

Funding Health and Medical Research Fund (Ref. No. 11121091), Hong Kong Special Administrative Region, China.

\section{6d WHAT SHOULD WE TELL SHIFT WORKERS TO DO TO REDUCE THEIR CANCER RISK?}

Damien McElvenney. Institute of Occupational Medicine, Edinburgh, UK

\subsection{6/oemed-2018-ICOHabstracts. 1378}

Introduction In 2007, a working group of the International Agency for Research on Cancer classified shift work that involves circadian rhythm disruption as probably carcinogenic to humans. We carried out a review of the human epidemiological and mechanistic evidence, along with relevant health and safety policies and practice, to identify practical measures that should be undertaken to reduce the risk of cancer among shift workers.

Methods A literature search was carried out of the Proquest Dialogue databases, which includes MEDLINE, in order to identify the relevant scientific literature published from 2005-2015. In addition, we carried out an internet search for current health and safety policies and practices for managing shift workers.

Results There is evidence from the epidemiological studies that the increase in breast cancer risk amongst women who have worked night shifts is relatively modest and could be zero. Other risk factors for breast cancer associated with night work, such as poor diet, should be addressed by employers. Evidence for other cancers remains limited and inconsistent. If a mechanism exists, it is most likely to be night-time suppression of melatonin production. There has been limited evaluation of interventions to manage shift workers and research in this area is fragmented. Health and safety policies provide little on prevention.

Conclusion Health and safety practices for shift workers should address reduction of health risks by choosing shift rotations that minimise impact on circadian disruption, by employers facilitating female workers to attend breast cancer screening and by promoting and facilitating a healthy lifestyle.

\section{6e CURRENT PROGRESS AND STATE OF SCIENCE IN POPULATION STUDIES OF CIRCADIAN/SLEEP DISRUPTION ON CANCER RISK AND SURVIVAL, AND CIRCADIAN PHASE BIOMARKERS}

\begin{abstract}
1,2Schernhammer Eva. 'Brigham and Women's Hospital and Harvard Medical School, Harvard School of Public Health, Channing Division of Network Medicine, Boston, MA 02115, USA; ${ }^{2}$ Department of Epidemiology, Medical University of Vienna, Austria
\end{abstract}

\subsection{6/oemed-2018-ICOHabstracts. 1379}

Melatonin (5-methoxytryptamine) is an indoleamine produced primarily by the pineal gland, which is secreted exclusively during the dark phase of the light-dark cycle in humans. Several decades ago, reports indicated that melatonin possesses oncostatic properties, leading to novel hypotheses that diminished secretion of melatonin might promote the development of cancer. Growing evidence also demonstrates that visible light, including electric light, can acutely suppress melatonin production- a phenomenon often referred to as 'circadian disruption' particularly if it occurs at night, as commonly observed in shift workers.

In 2007, the International Agency for Research on Cancer classified shift work as a possible carcinogen, based on convincing experimental evidence and supportive, but still limited, epidemiologic data. Indeed, experimental data has consistently demonstrated that circadian disruption can promote carcinogenesis in animals; specifically, exposure to light at night and phase shifts in the light-dark cycle have accelerated tumour development in rodents. In humans, epidemiologic data continues to accumulate, with the majority of existing studies indicating that shift work is related to a modest increase in the risk of breast cancer. Initial studies have identified links between shift work and other cancers as well, although this evidence is very limited.

In this presentation, I will review epidemiologic studies of circadian disruption/sleep and cancer risk, including some preliminary studies on cancer survival, and with additional emphasis on circadian phase biomarkers (melatonin/cortisol).

\section{SHIFTWORK IN THE 21ST CENTURY: OLD AND NEW PROBLEMS AND APPROACHES}

${ }^{1}$ Frida Marina Fischer*, ${ }^{2}$ Stephen Popkin. 'Department of Environmental Health, School of Public Health, University of São Paulo. São Paulo, SP, Brazil; ${ }^{2}$ U.S. Department of Transportation, Volpe Centre, Cambridge, USA

10.1136/oemed-2018-ICOHabstracts. 1380

Aim of special session This session is expected to be interactive, providing the attendees with both an understanding of the social and physical harm than may come with working shifts, and an evaluation of different countermeasures developed to help ameliorate these problems. The question and answer portion is meant to be both 'ask the expert' for those 